\section{Multisensor Data Fusion and Decision Support for Airborne Target Identification}

V.V.S. Sarma, Senior Member IEEE, and Savithri Raju

\begin{abstract}
A knowledge-based approach and a reasoning system for multisensor data fusion (MSF) is presented. The scenario taken for the example is an air-land battlefield situation. A data fusion system obtains data from a variety of sensors. This is an essential step in a Command, Control, Communication and Intelligence $\left(\mathrm{C}^{3} \mathrm{I}\right)$ system. Automatic processing of sensor data has become essential due to the volume of evidence available in real-time and to support higher level decision making processes. When several varieties of sensors are involved in the process of fusion, each contributing information at its own level of detail, we need to have a way to combine uncertain information from these disparate sensor sources at different levels of abstraction. Dempster-Shafer approach to represent and combine data is found appropriate for this, as this offers a way to combine uncertain information from several sources, each contributing in their own way. Evidential reasoning allows confidences to be assigned to sets of propositions rather than to just $N$ mutually exclusive propositions. The software has been developed in LISP language and tested on the IBM personal computer. The results illustrate the advantages of using multiple sensors in terms of increase in detection probability, increased spatial and temporal coverage and increased reliability that are very important in a battle-field/airdefense/naval-warfare situation.
\end{abstract}

\section{INTRODUCTION}

Every Command and Control $\left(\mathrm{C}^{2}\right)$ application employs a variety of sensors (e.g., radar, sonar, infrared (IR) detectors, seismometers) and sources (e.g., humint, photoint, data linked reports) to collect in formation necessary to develop a perception of the military situation. With the $\mathrm{C}^{2}$ application, fusion requirements vary, this is because the sensors have unique characteristics [2], [5], [7]. The parameters that characterize individual application are

1) Target quantities and categories that influence the size of the target data base and processing requirements.

2) Detection and decision rate requirements.

3) Number of sensors in the net and variety of sensors greatly influence the architecture and processing requirements on the data fusion algorithm.

\section{A. $C^{3} I$ Systems}

The phrase Command, Control and Communications evolved in the U.S. Department of Defense. In a military context, the phrase encompasses a range of operations and equipment: surveillance against suspicious or hostile acts; wartime and peacetime communications; all military satellites; radio and radar; jammers and countermeasures; and navigation equipment. Therefore, a military command and control system is a decision making network that reflects a hierarchical organization of command and control nodes. Within a $\mathrm{C}^{2}$ system, both command and control decision making occurs at every level of the hierarchy. Referring to Fig. 1 , the basic elements of a $\mathrm{C}^{3}$ system are:

Manuscript received August 10, 1990; revised March 1, 1991. A preliminary version of this paper was presented at the Workshop on Signal Processing, Communications and Networking, Bangalore, India, 23-26 July 1990.

V.V.S. Sarma is with the Department of Computer Science and Automation, Indian Institute of Science, Bangalore 560012, India.

S. Raju is with Electronics and Radar Development Establishment, DRDO Complex, CV Raman Nagar, Bangalore 560093, India.

IEEE Log Number 9102009

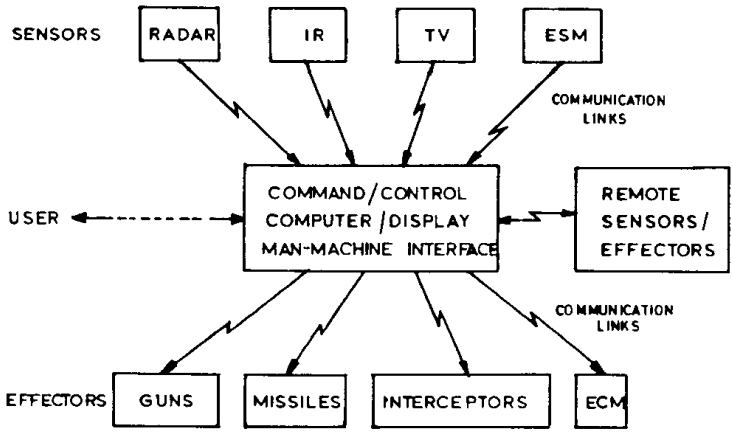

Fig. 1. Schematic representation of a complex air-defense system.

1) Sensor subsystems - to gather information about the location, movement and activities of enemy and friendly assets,

2) Navigation subsystems,

3) Command and fusion centers, and

4) Communication links.

Data fusion uses a combination of geographically distributed sensors of several types and multiple intelligent sources to collect information from these sensors and to develop the best possible perception of the military situation. The information processing includes:

1) Associating events of target detection to creating/ confirming target tracks or starting a new track corresponding to a different target, and

2) Detecting the presence and location of threat emissions. Identity of the targets as friend or foe can be achieved by a combination of the sensor outputs. Behavior of the targets are temporal (velocity, acceleration, course, etc.) and tactical activities (hostile acts such as jamming and other deceptive or engagement actions and friendly acts such as responding to IFF interrogation).

Benefits of multiple sensor data fusion in this scenario [1].

1) Robust Operational Performance: One sensor can contribute information while others are unavailable, denied or jammed or lack coverage of a target.

2) Extended Spatial Coverage: One sensor can look where another cannot.

3) Extended Temporal Coverage: One sensor can detect a target when others cannot.

4) Increased Confidence: More than one sensor to confirm the same target.

5) Reduced Ambiguity: Joint information from multiple sensors reduces the set of hypotheses about the target.

6) Improved Detection: Integration of multiple measurements of the target increases the assurance of the detection.

7) Enhanced Spatial Resolution: Multiple sensors can geometrically form a synthetic aperture capable of greater resolution than a single sensor can achieve.

8) Improved System Reliability: Multiple sensors suites have an inherent redundancy.

9) Increased Dimensionality: A system employing different sensors to measure various portions of the electromagnetic spectrum is less vulnerable to disruption by enemy action. 
To get the best possible perception of the military situation, data from several sources can be combined either as a single shot or as a continuous operation. Data fusion is not usually the end in itself but it is often an essential step in a command and control system. The results produced by data fusion can be used directly for control or to support situation assessment and decision making processes.

The scenario under consideration is an air-land battlefield situation. The targets include friendly and enemy aircraft, including fighters and bombers. Detection and identification of these targets are achieved by $\mathrm{RF} / \mathrm{IR}$ emissions, and radar echoes reflected from them. The sensors employed are as follows.

1) Radars located on the ground, which detect and locate air targets at slant ranges extending well beyond the forward line of own troops (FLOT).

2) Ground-based radar warning receivers (RWR) to detect the hostile emissions from air targets.

3) Infrared search and track sensor (IRST)

4) Information friend or foe sensor (IFF).

\section{B. Problem Formation}

Consider the sensor set $S$ given by

$$
S=\left\{s_{1}, s_{2}, \cdots, s_{M}\right\} .
$$

Each element in the set is a group of sensors of a different type. Each group can be considered as a set, that is:

$$
\begin{gathered}
s_{1}=\left\{s_{11}, s_{12}, \cdots, s_{1 n}\right\} . \\
\cdots \\
s_{M}=\left\{s_{M 1}, s_{M 2}, \cdots, s_{M n}\right\} .
\end{gathered}
$$

There are $M$ locations of the sensors and there are $n$ sensors in each group. Here $s_{i}$ refers to the sensor group at location $i$, $i=1,2, \cdots, M$ and $s_{i j}$ refers to the sensor of type $j, j=1,2, \cdots, n$ at location $i$.

Consider the target set

$$
T=\left\{t_{1}, t_{2}, \cdots, t_{x}\right\} .
$$

The unknown target is assumed to belong to a set of $N$ (known) targets belonging to this set, Target $t_{i}$ denotes the $i$ th target type. Each sensor output is processed at a sensor location to extract some characteristic features of the target and this data is sent to a fusion center to perform target detection and target parameter estimation [9]. Three general schemes exist for fusing information from sensors [10]. They are fusion of observations, fusion of decisions and fusion of probabilities. In this correspondence, we use the third scheme. Each sensor output is then a probability referring to the amount by which we believe in the proposition "Target $t$, detected by sensor $s_{i j}$ at range $R$, moving with a velocity $V$." We fuse this information with our belief in a similar proposition with detection by sensor $S_{i^{\prime} j^{\prime}}$, $i \neq i^{\prime}$ and $j \neq j^{\prime}$. We use Dempster-Shafer reasoning to perform this fusion [3], [8]. Let $z$ be an indicator function with $(z=1)$ denoting the presence of a target and $(z=0)$ denoting its absence.

Each sensor $s_{i j}$ is coupled with its own processor that maps an observation vector $y_{i j}$ into the detection probability $p_{i j}$ given by

$$
P_{i j}=P\left(z=1 \mid y_{i j}\right)=H_{i j}\left(y_{i j}\right) .
$$

A local fusion rule $H_{i}$ at a given location $i$ fuses the detection probabilities as given by

$$
\begin{aligned}
p_{i} & =P\left(z=1 \mid p_{i 1}, p_{i 2}, \cdots, p_{i n}\right) \\
& =H_{i}\left(H_{i 1}\left(y_{i 1}\right), \cdots, H_{i n}\left(y_{i n}\right)\right) .
\end{aligned}
$$

A global fusion rule $H$ gives the final detection probabilities as

$$
p=P\left(z=1 \mid H_{1}, H_{2}, \cdots, H_{M}\right)=H\left(H_{1}, H_{2}, \cdots, H_{M}\right) .
$$

\section{DECISION SUPPORT SYSTEMS FOR MULTISENSOR DATA FUSION}

The various types of factual or procedural knowledge can be combined to aid in target identification and parameter estimation. Expert system methods can be applied to fuse multisensor data by applying sets of rules directly to the measurements or derived parameters, as an alternative to classical statistical methods. Expert system methods may also find utility in optimally coupling the classification process with the positional estimation process.

\section{A. Why a Knowledge-Based Approach?}

Automatic processing of sensor data has become essential in order to cope with the volume of evidence available in real-time and to support higher level decision making. Battle management systems or combat decision aids require the interaction of data fusion and decision support functions. The challenge for the fusion system is to capture the knowledge of human experts in the machine's knowledge base.

Knowledge Representation by Rules: A set of facts can be organized and a set of rules can be stated such that if all of these facts represent true state, then the result is a conclusion that should be flashed to the operator. Expert or knowledge-based systems have dominated the use of AI technologies for military purposes. But still there are lot of unresolved issues in exploiting this technology fully. Automated fusion processing exhibits the following characteristics, not generally associated with first generation expert systems:

1) Time varying dynamic input states,

2) Real-time operational requirements,

3) Diverse data and knowledge classes,

4) Processing and message passing delays,

5) Spatial distribution of sensors,

6) Lack of human expertise in certain subproblem domains,

7) Multiple levels of abstraction in the decision process,

8) Large distributed knowledge bases.

Evidential Reasoning: This has recently received attention as an alternative to Bayes reasoning. This was originally conceived by Dempster and further developed by Shafer. By using this approach to represent and combine data, each sensor is allowed to contribute information at its own level of detail. Shafer-Dempster reasoning is a generalization of Bayes reasoning that offers a way to combine uncertain information from disparate sensor sources with different levels of abstraction.

Bayes theory offers a highly formalized and rigorous way to assign and propagate confidences. But, with reference to the problem of data fusion, there are several limitations in the application of Bayes theory. Bayes theory forces each sensor to respond with a Bayesian family of beliefs over a common level of abstraction. An example on this is in [3]. If the evidences are conflicting then Dempster-Shafer rule cannot be applied straightaway, one has to adopt the modified form of Dempster-Shafer reasoning as suggested in [8].

\section{B. Sensors}

The outputs of the sensors, described under introduction (scenario) and Section I-B are to be fused using Dempster-Shafer theory of evidential reasoning. The different stages of the work are described in the following. 
Simulation of Track Knowledge Base: We need to simulate a track knowledge base, which is a dynamic input to the data fusion system and is diverse in nature. Alpha Beta tracking filter algorithm is used for smoothing and prediction. The outputs are tracks: the smoothed values of position, velocity and acceleration at a series of points in the trajectory, and the predicted values of position and velocity.

Introduction to Filtering: Filtering and prediction are the fundamental elements of any tracking system. Filtering and prediction methods are used to estimate present and future target kinematic quantities such as position, velocity and acceleration. Two commonly used approaches to filtering and prediction are to use the fixed tracking coefficients and variable tracking coefficients that are determined by a priori models for the statistics of measurement noise and target dynamics. Kalman filtering is an example of this. Both of these types of filters are of fading memory type, which can be implemented recursively. Data received in the past are included in the present estimate, and hence all data are utilized, but forgotten at an exponential rate. The term "smoothed" as used in the literature of fixed coefficient filtering is synonymous with the term "filtered," which is used in Kalman terminology.

Fixed Coefficient Filtering: Fixed coefficients have the advantage of simple implementation using fixed parameters for the filter gains. The most extensively applied fixed coefficient filter is the Alpha Beta tracker. This filter is used when only position measurements are available, and is defined by the following equations:

$$
\begin{aligned}
X_{s}(k) & =X_{p}(k)+\alpha\left[X_{0}(k)-X_{p}(k)\right] \\
V_{s}(k) & =V_{s}(k-1)+\beta / q T_{s}\left[X_{0}(k)-X_{p}(k)\right]
\end{aligned}
$$

where $X_{0}(k)$ is the observation received at $k, X_{s}(k)$ and $X_{p}(k)$ are respectively the corresponding smoothed and predicted values, $\Gamma_{s}(k)$, $V_{s}(k-1)$ are the smoothed velocities at the instants $k$ and $(k-1)$ respectively. $T_{s}$ is the sampling interval. $a, \beta$ are fixed coefficient filter parameters. The quantity $q$ is defined to be unity.

There are four cases of acceleration of the target dealt with.

1) LACC $=0$, RACC $=0$ (Both linear (LACC) and Radial (RACC) acceleration are 0 ).

2) $\mathrm{RACC}=0, \mathrm{LACC} \neq 0$ (Constant linear acceleration).

3) $\mathrm{RACC} \neq 0, \mathrm{LACC}=0$ (Constant radial acceleration-circular turn(].

4) $\mathrm{RACC} \neq 0, \mathrm{LACC} \neq 0$ (Both linear and radial acceleration are nonzero).

From the target course (CT), conventionally measured in degrees clockwise with respect to north, and LACC and RACC values taken from the input data base file, the accelerations in the $X$ and $Y$ directions XDDT and YDDT are computed.

Target position and velocity updating are done using the starting position, scan time, LACC, RACC, course etc., values extracted from the input data files. In the simulation, radar data is corrupted with noise. The noise has Gaussian distribution with zero mean and standard deviation SXM, SYM in the $X$ and $Y$ directions. The smoothed position, velocity and acceleration in the $X$ and $Y$ directions are then determined. The algorithm chooses the input values from the data input file. The trajectory can be divided into segments, and for each segment, linear, radial accelerations, time of dwell are taken from the input file.

Outputs of this filtering program are a series of smoothed position, velocity and acceleration values in $X$ and $Y$ directions, and a series of predicted position, velocity values in the $X$ and the $Y$ directions, for the tracks. This information is stored in a text file. This forms the simulated track knowledge base.

For each of the radar sensors, a few tracks are generated through simulation and stored in the frame structure that is used in correlation.
For simulation, the input data are chosen from the data base file through uniform random number generation. A typical example is the following, which is a frame structure implemented as nested association list in LISP.

$$
\begin{aligned}
\text { (Setc Track1 (List ' } & ((\text { Sposinx (Value }(54083.4))) \\
& (\text { Sposny (Value }(36833.6))) \\
& (\text { Srelox (Value }(-59.4))) \\
& (\text { Sreloy (Value }(-369.4))) \\
& (\text { Saclnx }(\text { Value }(8.6))) \\
& (\text { Saclny (Value }(-9.9))) \\
& (\text { Predx }(\text { Value }(54072.3))) \\
& (\text { Predy (Value }(36763.2))) \\
& (\text { Predxd (Value }(-57.7))) \\
& (\text { Predyd (Value }(-371.3))))) .
\end{aligned}
$$

The smoothed values of position, velocity, acceleration and the predicted values of the position, velocity are stored as in the previous structure. No multiple target tracking is done here.

In the simulation, only radar sensors on the platforms are considered first. Correlation of the incoming radar returns with the existing tracks is tried. If the report does not correlate with any of the tracks, a new track is initiated based on $3 / 4$ logic. That is, if out of four consecutive scans, three of the reports are from the same target, then the target is confirmed. After the track is confirmed, it is added to the existing track knowledge base. The evidences are then combined.

As the next step, more sensors are considered. For any detection, if there is more than one sensor in a platform that can detect it, their evidences are combined. The outputs of this stage are individual platform supports, which are combined at the central data fusion center to give the overall support and uncertainty values.

Referring to Fig. 2, the blocks designated plat 1, 2, and 3 are platforms. Data inputs to the system, which are the outputs of the various sensors are chosen randomly from data input files. For simulation, random number seeds are input at the time of program execution. Having chosen data inputs, it is verified whether the target can be detected at this range based on the location of the sensors, and the corresponding probability of detection is determined in the case of radar sensors, from the stored knowledge about the probability of detection versus the signal to noise ratio for a fixed probability of false alarm. In the case of RWR and IRST sensors, the results of a simulation work of Bogler [3] are prestored as the sensor knowledge base in the form of look up tables. From this, the average confidence in detection is got. In the case of IFF sensor, it is assumed that, if an IFF interrogation elicits a response, then probability of the target being a friend $(\mathrm{P}(\mathrm{F})=1.0(\mathrm{~F}=$ Friendly $))$. If the IFF does not respond, this means that chances are more for the target to be hostile, hence probability of the target being hostile $(\mathrm{P}(\mathrm{H})=0.8 .(\mathrm{H}=$ hostile $))$ and we are uncertain to an extent of $20 \%$, which we would assign to ignorance. That is, the probability of uncertainty, $P$ (uncertainty $))=0.2$. Uncertainty is designated by $\theta$. We also perform the target tracking and data correlation/association in this step.

Data Combination/Fusion: Evidential reasoning is used for data fusion. Here the evidences provided by each of the sensors on a single platform, are combined. If a target falls in the intersection of the coverages of two radars, the corresponding supports are combined at the central fusion center. 


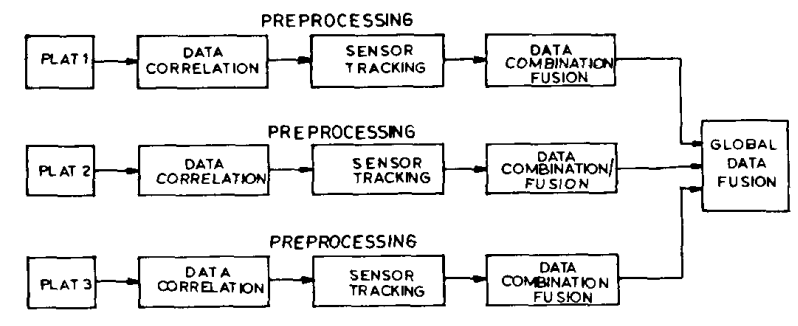

Fig. 2. System block diagram.

\section{IMPLEMENTATION OF KNOWLEDGE-BASED DATA Fusion}

\section{A. Sensor Knowledge Bases}

Sensor knowledge bases can be categorized into the following for the radar sensor:

Parametric Knowledge: This is the knowledge about the radar sensor parameters such as transmitter power, antenna gain, maximum range detectable, and altitude maximum etc. Frame structure, which can be implemented as nested association lists in LISP is a convenient form to represent this knowledge. With this form of representation, it is easy to plug in the individual values of sensor characteristics or parameters in a frame using a simple function in LISP:

$$
\begin{aligned}
\text { Eg. (Setq Radar1 } & \text { (Maxrange (value }(120)) \\
& (\text { Height (value }(70))) \\
& (\text { Tx_power (value }(---)))) .
\end{aligned}
$$

Also stored are the parameters such as the distance of one platform from the other, in both $X$ and $Y$ directions, and the receiver bandwidth.

Performance Knowledge Base: The probability of detection versus the signal to noise ratio, for a given probability of false alarm is the appropriate knowledge for describing the performance of a radar sensor. This is stored as association list.

Simulated Knowledge Base: The knowledge about the targets already detected and their tracks is included in this category. For simulation of tracks, Alpha Beta filtering algorithm is used. This knowledge base is stored in a frame structure.

\section{B. RWR Sensor Knowledge Base}

This sensor is a passive sensor giving early warning about threats. Associated with this sensor, we need a threat library. Threat library is stored in the form of a frame. This contains the hostile frequencies, pulse width and pulse repetition frequencies for several values. The detected range versus the average confidence in the detection corresponding to this range obtained via simulation is stored in a nested association list form.

An example is the following:

$$
\begin{aligned}
(\text { setq hostf } & ((\text { freq }(\text { value }(6.9))) \\
& (\text { pw }(\text { value }(200))) \\
& (\text { prf }(\text { value }(100))))) .
\end{aligned}
$$

Here we have set hostile frequency (hostf) equal to $6.9 \mathrm{GHz}$, pulse width ( $\mathrm{pw}$ ) equal to $200 \mathrm{~ns}$ and pulse repetition frequency (prf) equal to $100 \mathrm{~Hz}$. The range versus the corresponding average confidence are stored in association list format. Once a target detection occurs, the corresponding average confidence factor is plugged out from this knowledge base.

\section{IRST Sensor Knowledge Base}

Similar to the RWR, for this sensor, the average confidence values are stored.

\section{Data Bases}

For each of the sensors we need to simulate the inputs. These are stored in an appropriate form in files, and chosen randomly. The different data are:

1) For Radar Sensor: The position, velocity of the target and the sensor identification that detects one target are stored. By choosing a corresponding index, these data can be accessed. This index is generated randomly through uniform random number generation. We choose the same index to represent the same target, which means that the same random number is chosen for selection of data input for various sensors.

2) RWR and IRST Sensors: The inputs of these sensors are also stored in data file, in frame format.

3) IFF Sensor: IFF interrogation elicits a response if the target is friendly, and no response implies that the target is hostile or IFF in the target is malfunctioning. We assume in this case that the probability of the target being hostile is high say $80 \%$.

\section{E. Implementation of Data Fusion}

This is carried out in two stages. In the first stage, only radar sensors are considered on each of the platforms. In the second stage, all other sensors are also considered.

Stage 1: When a target is detected, depending on its relative location with respect to the radar, we can determine which other radar can detect it. The coverage of the radar sensors on platforms 1 and 2 can overlap, so also the coverage of the radars on platforms 2 and 3. In other words, there is a common area where two of the radars can cover. If a target falls in the intersection of radars 1 and 2 then a predicate FALL12 is set to true. If it falls in the intersection of 2 and 3 then predicate FALL23 is set to true. If neither FALL12 or FALL23 is true, this case is treated differently. Now we have to deal with two cases:

1) Single radar detecting a target and

2) More than one radar detecting it.

We determine the probability of detection and corrupt the data with Gaussian noise. Correlation of this with the existing track knowledge base is tried out. Finally, the data are fused, if more than one evidence is available.

From the target position in the $X$ and $Y$ direction, we get the slant range. Set FALL12 or FALL23 as appropriate. Then determine the signal to noise ratio for this range. Using the radar range equation and the radar sensor parameters from the knowledge base file, we get the signal to noise ratio for this range. Probability of detection can be treated as a confidence factor or evidence in the proposition "Target $T_{i}$ has been detected at range $r_{i}$."

Based on whether one or more than one radar is able to detect the target, we either fuse the evidences and display the support and uncertainty values or display only the single radar's evidence as the support. Therefore, the outputs of this phase are the support and uncertainty values.

2) Implementation of Data Fusion for a Multiple Sensor Scenario: The results and principles of stage 1 , wherein we considered three radars on three individual platforms can be modified to accommodate more numbers of sensors. A few assumptions made are as follows:

1) At any time, only one sensor in the net is jammed,

2) The RWR, IRST, and IFF sensors can detect targets at the ranges that the radar sensor can detect, 


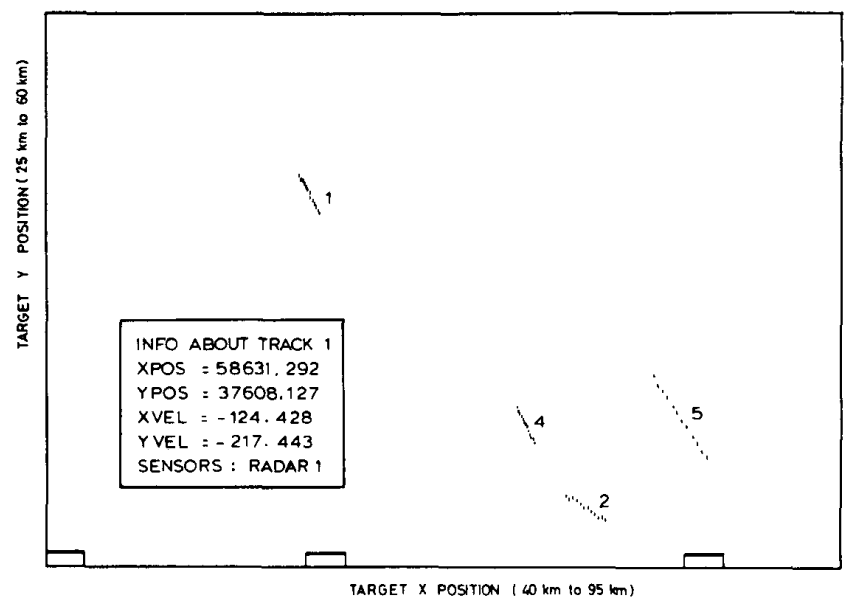

Fig. 3. Target tracks and fused information.

3) We choose the same seed for random selection of the data input, so that the same target is chosen for analysis and

4) For the sensor that is jammed, the corresponding confidence it provides is zero.

With these assumptions we proceed as follows: Choose randomly an input from the data input file. Based upon the sensors that can detect it, the corresponding evidences are computed. If the radar misses the target, we set a predicate RADARDET to $N$ else to $Y$. Three different cases are considered:

1) All four sensors involved in fusion.

2) Three sensors involved: Radar, RWR and IFF, or Radar, IRST, IFF, etc. (random occurrence).

3) Two sensors involved: Radar and IFF (random occurrence).

Example 1: An example of fusing the evidences provided by the sensors is given in the following: Consider the sensors Radar1, Radar2, RWR, and IFF, the corresponding evidences being

$\mathrm{P} 11($ det by Radar $)=0.96$ (probability of detection by Radar1)

$$
\operatorname{P} 11(\theta)=0.04
$$

$\mathrm{P} 12($ det by Radar $)=0.56$ (probability of detection by Radar2)

$$
\begin{aligned}
\mathrm{P} 12(\theta)= & 0.44 \\
& \text { by the RWR) } \\
\mathrm{P} 2(\theta)= & 0.62 \\
\mathrm{P} 3(\mathrm{~h})= & 0.8 \text { (this is by IFF sensor) } \\
\mathrm{P} 3(\theta)= & 0.2
\end{aligned}
$$$$
\mathrm{P} 2(\mathrm{~h} \text { emit })=0.38 \text { (This is the evidence provided }
$$

where det means detection, hemit means hostile emission. Now, let us combine these using Dempster's rule of combination. Combining $\mathrm{P} 2$ and $\mathrm{P} 3$ we get fused values as in Table I. Therefore $\mathrm{P}(\mathrm{h}$ emit $)=0.876(=0.304+0.496+0.076)$. That is the probability of target hostility has increased from 0.8 to 0.876 . In this case IFF is the major sensor and RWR is a supporting one.

Consider now the detection probabilities of radars for combination. Combining P11 and P12 we get fused outputs as in Table II. Therefore $\mathrm{P}($ det $)=0.9824$ and $\mathrm{P}(\theta)=0.0176$.
TABLE I

Fusion Results fOR SENSORS P2 AND P3

\begin{tabular}{lll}
\hline P2 (h emit $)=0.38$ & $\mathrm{P}($ h emit $)=0.304$ & $\mathrm{P}(\mathrm{h}$ emit $)=0.076$ \\
$\mathrm{P} 2(\theta)=0.62$ & $\mathrm{P}(\mathrm{h})=0.496$ & $\mathrm{P}(\theta)=0.124$ \\
& $\mathrm{P} 3(\mathrm{~h})=0.8$ & $\mathrm{P} 3(\theta)=0.2$ \\
\hline \multicolumn{3}{c}{ TABLE II } \\
\multicolumn{3}{c}{ FUSION RESULTS FOR SENSORS P11 AND P12 } \\
\hline $\mathrm{P} 11($ det $)=0.96$ & $\mathrm{P}($ det $)=0.5376$ & $\mathrm{P}($ det $)=0.4224$ \\
$\mathrm{P} 11(\theta)=0.04$ & $\mathrm{P}($ det $)=0.0224$ & $\mathrm{P}(\theta)=0.0176$ \\
& $\mathrm{P} 12($ det $)=0.56$ & $\mathrm{P} 12(\theta)=0.44$ \\
\hline
\end{tabular}

Thus, we see that the detection probability increases from 0.96 to 0.9824 , with the target being hostile, and uncertainty reduces to 0.0176 from 0.04 .

If sensors situated in more than one platform, detect the target, then their evidences are in turn fused. First fuse the evidences provided by sensors in a particular platform, which gives the platform support. Next fuse the platform supports. A few test data and results are attached in Appendix A. Sample target tracking results are shown in Fig. 3.

\section{CONCluSion}

This correspondence presents an exploratory study of multisensor data fusion and the example illustrates the implementation of a system to fuse data. The real role of data fusion is that of converting data into meaningful and timely concept information that will enhance the process of human judgement while making a critical battlefield decision. The current concerns that such automated systems will be vulnerable to deception are based on the premises that countermeasures and tactics often rely on unpredictable behavior. The role of decision support system is to quantify this uncertainty to help the human commander to cope with these higher order reasoning processes in the hostile environment of a battlefield while facing an intelligent adversary.

APPENDIX IMPLEMENTATION RESULTS

There are two choices for the user and the system is initiated. A typical session of the program is as follows: 


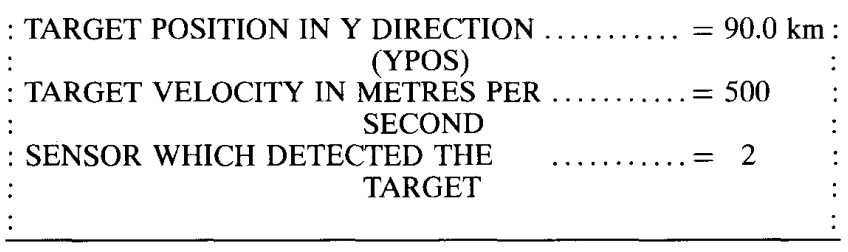

READ U1, U2, U3 REAL NUMBERS SAME AS YOU READ IN JUST NOW TO SELECT

SAME INDEX

$3.3 \quad 3.3 \quad 3.5$

RWR HAS NOT DETECTED THE TARGET

IFF RESPONSE IS : POSITIVE

IRST IS NOT ABLE TO DETECT THE TARGET

HEIGHT $=91.1172$

GROUND RANGE $=88.8687$

RADAR SENSOR 3 HAS BETTER PROBABILITY

RADAR SENSOR 3 IS THE MAJOR ONE \& RADAR SENSOR 2 SUPPORTS IT

HOW MANY SENSORS HAVE BEEN JAMMED ?

0

GOOD LUCK ! NONE OF THE SENSORS HAVE BEEN JAMMED

NONE OF THE SENSORS IN PLATFORM NO 2 IS JAMMED

READ UR1, UR2, UR : BETWEEN 0 AND 1 : SEEDS FOR UNIFORM RANDOM NUMBER GENERATION

FOR CORRUPTING INPUT DATA WITH NOISE

0.30 .40 .6

NO OF TIMES $=1$

THIS IS THE CASE OF 2 SENSORS :RADAR \& IFF NONE JAMMED

IFF CONFIDENCE $=1.0$

EVIDENCE BY RADAR $2=0.11$

EVIDENCE BY RADAR $3=1$

THIS IS CERTAINLY A FRIENDLY TARGET WITH $100 \%$ SUREITY

SUPPORT BY RADAR SENSORS $=1.0$

UNCERTAINTY $=0.0$

\section{REFERENCES}

[1] E. L. Waltz and D.M. Buede, "Data fusion and decision support for command and control," IEEE Trans. Syst., Man, Cybern., vol. SMC-16, pp. 865-879, 1986.

[2] C. J. Hatris, Ed., Application of Artificial Intelligence to Command and Control Systems. London: Peter Peregrinus, 1988.

[3] P. L. Bogler, "Shafer-Dempster reasoning with applications to multisensor target identification systems," IEEE Trans. Syst., Man, Cybern., vol. SMC-17, pp. 968-977, 1987.

[4] Reiner, "Application of expert systems to sensor fusion," Nat. Aerosp. Electron. Conf. (NAECON) record, vol. 2, pp. 1444-1450, 1985.

[5] D.C. Schleher, Introduction to Electronic Warfare. Washington, DC:
Artech House Inc., 1986

[6] G. B. Wilson, "Some aspects of data fusion," Proc. IEEE Conf. Advances in Command, Control and Commun. Syst., 1985, pp. 99-104.

[7] A. Farina and F. A. Studer, Radar Data Processing, vol. 1. Letchworth, England: Research Studies Press Ltd., 1986.

[8] H. Y. Hau and R. L. Kashyap, "Belief combination and propagation in a lattice-structured inference network," IEEE Trans. Syst., Man, Cybern., vol. 20, No. 1 , pp. $45-58,1990$.

[9] K. Demirbas, "Distributed sensor data fusion with binary decision trees," IEEE Trans. Aerosp. Electron. Syst., vol. 25, pp. 643-649, 1990.

[10] R. Krzysztofowicz and D. Long, "Fusion of detection probabilities and comparison of multisensor systems," IEEE Trans. Syst. Man Cybern., vol. 20 , no. 3, pp. $665-677,1990$. 